\title{
Cauda Equina Syndrome-Induced Acute Urinary Retention in Patients with Acute Lumbar Disc Herniation: Report of Two Clinical Cases.
}

Hoai Thi Phuong Dinh ( $\sim$ phuonghoai1412.md@gmail.com )

Hue University of Medicine and Pharmacy - Hue University https://orcid.org/0000-0002-9807-5953 Hai Minh Nguyen

Duyen Nha Le

Anh Thi Quy Le

Minh Thanh Nguyen

Hung Khoa Nguyen

\section{Case report}

Keywords: Cauda equina syndrome, acute urinary retention, saddle anaesthesia, lumbar disc herniation

Posted Date: August 31st, 2020

DOI: https://doi.org/10.21203/rs.3.rs-63647/v1

License: (c) (i) This work is licensed under a Creative Commons Attribution 4.0 International License.

Read Full License 


\section{Abstract}

Objective: to discuss two cases with CES-induced acute urinary retention, knowledge of which is essential for early diagnosis, treatment and prevention of neurological dysfunctions.

Case presentation: Two patients were hospitalized with symptoms lower back pain with weakness and progressive loss of feeling of lower limbs accompanied by acute urinary retention, urinary system tests were within normal limits. Magnetic resonance imaging results in lumbar disc herniation causing spinal stenosis. Both patients underwent posterior lumbar interbody fusion (PLIF) procedure and showed improvements in symptoms after surgery and re-examination.

Conclusion: The diagnosis should be based on magnetic resonance imaging. Lumbar disc herniation is the most common cause of acute urinary retention from cauda equina compression. Laminectomy, with early decompression within 24 to 48 hours, reduces the risk of long-term neurological dysfunction and increases the chances of recovery for the patient.

\section{Introduction}

Cauda equina syndrome (CES) is a rare but devastating neurologic condition resulting from many causes. The most common cause of CES is the compression/herniation of a lumbar spinal disc at L4/L5 and L5/S1 level ${ }^{1}$, accounting for about $2 \%$ of lumbar disc herniation cases. It is also one of the emergency surgical indications ${ }^{2}$.

The mechanism of injury to the nerves in CES is due to direct mechanical pressure, inflammation, and venous congestion or ischemia ${ }^{3}$.

According to Gleave and Marfalanc, cauda equina syndrome is divided into two types: Incomplete and Complete. Type 1: incomplete cauda equina syndrome - CESI with partial control of urination (altered urinary sensation, loss of urgency, poor urinary stream and the need to strain in order to micturate). Type 2: cauda equina syndrome with retention - CESR with the bladder no longer under executive control (urinary retention or incontinence micturition) ${ }^{4}$.

Cause the cauda equina nerve roots control of the pelvic organs and the lower body, so CES presents acutely which some or all of the symptoms: The prevalence of bladder dysfunction was $76 \%$, bowel dysfunction $13 \%$, sexual dysfunction $39 \%$ and physical dysfunction $48 \%$. Patients presenting with CES-R had significantly worse long-term outcomes in bladder (stream domain), bowel and sexual function compared to those with CES-I ${ }^{5}$.

Magnetic resonance imaging (MRI) is the gold standard for the diagnosis of cauda equina syndrome and helps distinguish it from diagnoses such as aortic dissection or spinal infarction ${ }^{6}$. Myelography and CT (computed tomography) Myelography can be useful alternatives for patients who cannot have an MRI, but it is an invasive technique. Clinical diagnosis of cauda equina syndrome can have a false positive 
rate of $43 \%^{7}$. The most important issue associated with the outcome is time to early diagnosis and treatment, for the prevention of permanent neurological dysfunctions.

Therefore, we would like to discuss two clinical cases of acute urinary retention related to cauda equina syndrome in patients with acute lumbar disc herniation, to help clinicians gain quick assessment and appropriate management to avoid severe complications to the patient.

\section{Case Presentation}

\section{Case 1}

A 63-year-old man was brought to the hospital with weakness and loss of sensation of both lower limbs, progressing for 2 weeks, and lower back pain for 1 year with a recent increase in pain and urinary incontinence. The patient suffered from decreased muscle strength of 2 lower limbs, saddle anesthesia and decreased anal tone. He had a history of 3 years of hypertension regularly treated.

At the time of hospitalization, he was conscious and coherent. Laboratory data showed erythrocytes 3.7 T/ I, white blood cells $8.52 \mathrm{G} / \mathrm{I}$, platelet $188 \mathrm{G} / \mathrm{I}$, normal total urinalysis, liver and renal function with normal triglyceride, cholesterol, and uric acid levels. Serum creatinine was $6.2 \mathrm{umol} / \mathrm{L}$, and serum urea $8.3 \mathrm{mmol} / \mathrm{L}$. MRI scan (Fig. 1) reveals hypointensity of the disk stratum from the L3 level. At the level of L3-L4, there is a larger herniation downward, causing severe narrowing of the spinal canal (degree C), the foraminal zone on both sides, and compression of the cauda equina. At the L4-L5 level there is intervertebral disk protrusion (degree B), narrowing of the left foraminal zone, with the nerve root insertion not clearly visualized. At the L5-S1 level, there is central and lateral disc herniation and epidural compression, affecting the corresponding nerve roots in the right segment of the spinal canal. The diagnosis was made of cauda equina syndrome and a classical surgery ensued, with decompression at the L3-L4 level using the posterior lumbar interbody fusion (PLIF) procedure (Fig. 2). The postoperative period had no complications and the patient was discharged after 10 days with bilateral leg muscle function restored; however, sphincter dysfunction did not improve which necessitated intermittent catheterization. After 2 months, the patient was re-examined with motor function recovered almost completely, while the sensation of urination and defecation improved markedly. He could urinate after 3 months.

\section{Case 2}

A 33-year-old woman presented to the emergency department for low back pain and numbness in both lower limbs. Two weeks earlier, she experienced sudden pain in her lower back after having carried a heavy box. She was diagnosed with lumbar spinal degeneration and treated with acetaminophen and acupuncture for 3 days without relief. Four days earlier, she had numbness gradually increasing in both lower limbs, urinary retention (with urinary catheterization at a private clinic producing $400 \mathrm{ml}$ urine), and constipation for 3 days. 
On physical examination, the patient was alert and oriented with stable vital signs. The straight-leg-raise test to 30 degrees was positive on both legs. Motor strength of the left lower limb was decreased to 3 out of 5 in the hamstring and quadriceps muscles, and 2 to 3 out of 5 in the ankle dorsiflexor muscles and extensor hallucis longus muscle. Patellar reflexes were normal, but Achilles tendon reflexes decreased. Sensory examination demonstrated paresthesia of perianal area. The anal sphincter tone was reduced.

Lumbar spine radiographs revealed mild narrowing of the intervertebral disk spaces between L4-L5 and L5-S1. Magnetic resonance image (MRI) of the lumbar spine shows an extruded disc herniation at the L5S1, and a posterior protrusion at L4- L5 resulting in central canal stenosis and compression of the cauda equina (Fig. 3). The patient underwent emergency surgery. A central intralaminar approach was used at the L4-L5, L5-S1 interspace. The ligamentum flavum was then removed and an adequate width decompression was performed providing exposure of the dural sac using the posterior lumbar interbody fusion (PLIF) procedure (Fig. 4). The postoperative period was without complication, and sensory-motor function improved; the patient still had a hypotonic anal sphincter and no sensation of urination. She was referred to a rehabilitation unit for continuing therapy and instructed to do intermittent urinary catheterization. Two months later, the patient was able to walk normally, despite the wide and slow gait. The sensation of urination and defecation significantly improved 3 months after surgery.

\section{Discussion}

There are many causes of CES such as spinal stenosis, tumour, cysts, infection or bony ingress. However, lumbar disc herniation is the most common ${ }^{8}$ with compression/herniation of a large central lumbar disc at the L4-L5 and L5-S1 levels ${ }^{1}$.

The pathogenesis of CES has not been clearly defined. There is a hypothesis that large central or paracentral disc prolapses cause extrinsic compression of the lumbosacral nerve roots below the level of the Conus Medullaris. Nerve root compression, especially in a stenotic canal produce a congestion and dilation of intraradicular and periradicular vein, frequently observed intraoperatively and described as varix ${ }^{9}$. However, it is not clear what quantifies significant canal compression leading to CES. Another hypothesis said that CES is due to chemicals mediated with inflamed and oedematous neural structures being found on pathological samples ${ }^{10,11}$. Alternatively, CES was incriminated an autoimmune reaction 12 axonal demyelination with wallerian degeneration induced by TNF-a expression ${ }^{13}$

Cauda equina syndrome is caused by distal nerve root dysfunction and can be divided into incomplete CES (CES-I) and complete CES (CES-R). In complete CES, the patient has saddle anesthesia and bowel dysfunction that has progressed to full retention or incontinence. With incomplete CES there is also saddle anesthesia but the bladder and bowel dysfunction does not progress to urinary retention or incontinence ${ }^{14}$. At the beginning, they have difficulty in starting or stopping a stream of urine, after wards are present urinary retention or overflow incontinence ${ }^{9}$. As urinary retention is one of the important predictors of cauda equina compression, urinary retention of more than $500 \mathrm{ml}$ alone or in combination of two or more specific clinical characteristics were the most important predictors of MRI confirmed 
cauda compressions ${ }^{15}$. The striking feature is that in case 1 , the patient suffered a urinary incontinence, the patient in case 2 had a urinary retention with $400 \mathrm{~mL}$ urine taken out by catheterization. Therefore, both patients were classified as CES - R.

Magnetic resonance imaging (MRI) is the gold standard for diagnosing CES. MRI helps to clearly describe soft tissue pathology and to determine the extent of damage, as well as to help distinguish CES from other conditions such as aortic dissection or spinal infarction. The disadvantage of MRI is that it cannot be used in patients with pacemaker or poor tolerance due to claustrophobia ${ }^{6,16}$. Magnetic resonance imaging (MRI) is not as clearly defined as computed tomography (CT) but it is better at displaying soft tissues such as a disc, ligament, dural sac and nerve roots ${ }^{16}$. However, it is estimated that there are fewer than 1 in 2000 of patients presenting with acute severe lower back pain will be diagnosed with CES and up to $19-41 \%$ of patients referred for CES evaluation have essentially negative imaging studies 7 . Therefore, it requires a thorough workup to make an accurate diagnosis and a specimen be provided for pathological analysis is necessary to confirm the aetiology. Clinical diagnosis of cauda equina syndrome, even by resident neurosurgeons, has a false positive rate of $43 \%$, so imaging is important for diagnosis ${ }^{7}$.

Although CES is considered as a surgical emergency, the timing of surgical intervention remains controversial. Some studies suggest that early decompression within 48 hours is associated with better outcome. The operation within 24 hours may decrease the occurrence of abnormal urinary function while the operation within 48 hours may help to reduce usage of catheter ${ }^{17}$.

According to Dinning and Schaeffer, urinary disturbances improved to a greater degree in the patients who underwent decompression within 24 hours ${ }^{18}$. Nielsen's study indicated that detrusor function return was greatest in patients who had decompression within 48 hours of onset of symptoms ${ }^{19}$. Hellstrome et al. also reported improvements in the sexual potential following early surgery ${ }^{20}$. Similarly, Shapiro reported an improvement rate of $100 \%$ for urinary symptoms, when decompression surgery was conducted within 48 hours and $33 \%$ when surgery was conducted after 48 hours $^{21}$.

However, a meta-analysis of CES of Ahn et al. (2000) found no significant differences in outcomes between patients treated less than 24 hours after onset of CES and those treated within 24-48 hours ${ }^{1}$. The studies of Reddy, A. P. et al (2018), Heyes, G et al (2018), Delgado-López et al (2019) suggested that there were no statistically significant differences in functional outcome between patients operated within 48 hours and patients operated after 48 hours ${ }^{22-24}$. According to Xunwei Lai et al (2017), all of their patients received much delayed decompression (mean 4.1 weeks for CES - R and 5.5 weeks for CES - I) and $50 \%$ of the patients with CES - $\mathrm{R}$ achieved good recovery and the others also reported significant improve after surgery and all of the patients with CES - I had normal life after delayed decompression ${ }^{25}$

In our cases, both patients were sent to hospital and operated after 2 weeks from initial symptoms. The postoperative period was without complications and sensory - motor function improved; however, both 
patients required intermittent catheterization, and the rehabilitation of sphincter function and sensation of urinary and defecation lasted over 2 months after surgery.

Mugge et al (2019) reported 1 case of CES hospitalized after 3 months from the onset of symptoms. After the surgery, the patients complained of only incisional pain and he was discharged on postoperative day $3{ }^{26}$. Douraiswami et al (2016) reported a case of CES caused by disc prolapse. The decompression was done 18 days after the onset of symptoms and the patients showed complete recovery. However, all authors of this report still recommended surgery as soon as possible to maximize functional recovery, especially the bladder symptoms ${ }^{27}$.

Although there is no truly clear evidence of the association between the timing of surgery and the prognosis of sphincter function, we think that the surgery intervention should be performed as soon as possible in order not to miss the abilities for patients to recover.

Therefore, early decompression surgery for all cases is recommended. Clinical diagnosis should be determined as early as possible if symptoms suggest possible CES, and a lumbar spinal MRI should be performed. Coordination closely between many specialties such as Nephrology, Urology, and

Rehabilitation is needed to achieve the best recovery outcomes for patients. Neurological defects, such as neurological bladder disorders, have a major impact on the quality of life in patients after surgery and require a relatively long recovery period, from several weeks to several years. Intermittent catheterization is a safe and effective solution during this period of post-operation, greatly improving the quality of life and the effectiveness of treatment, so it is widely studied and applied to clinical practice. In our cases, both patients had the ability to urinate recover after 3 months, thanks to the rehabilitation combined with traditional medicine after surgery.

\section{Conclusion}

Cauda equina syndrome due to lumbar disc herniation is rare but causes severe complications. Clinical diagnosis may be easily confused with urological pathology even by experienced clinicians. The diagnosis should be based on magnetic resonance imaging. Acute urinary retention from cauda equina compression requires early surgical intervention with laminectomy within 24 to 48 hours, to reduce the risk of long-term neurological dysfunction and increases the opportunities of recovery for the patient.

\section{Declarations}

Authors declare no conflict of interest.

\section{Ethics approval and consent:}

Accepted by the ethical approval committee/Institutional Review Board of Hue University of Medicine and Pharmacy, No. 2379 / QD-DHYD, August 15, 2020. 
Consent for publication:

Obtain permission and consent from patients (attached form)

\section{Competing Interests:}

The authors declare that they have no competing interests

\section{Authors' contributions:}

Authors equally contributed the work. All authors read and approved the final manuscript.

\section{Acknowledgements:}

No

\section{References}

1. Ahn UM, Ahn NU, Buchowski JM, Garrett ES, Sieber AN, JP. K. Cauda equina syndrome secondary to lumbar disc herniation: a meta-analysis of surgical outcomes. Spine. 2000;25:1515-1522.

2. Dommisse GF. Morphological aspects of the lumbar spine and lumbosacral region. The Orthopedic clinics of North America. 1975;6 1:163-175.

3. Gleave JR, R. M. Prognosis for recovery of bladder function following lumbar central disc prolapse. Br J Neurosurg. . 1990;4:205-209.

4. Gleave JR, Macfarlane R. Cauda equina syndrome: what is the relationship between timing of surgery and outcome? British journal of neurosurgery. Aug 2002;16(4):325-328.

5. Hazelwood JE, Hoeritzauer I, Pronin S, Demetriades AK. An assessment of patient-reported long-term outcomes following surgery for cauda equina syndrome. Acta Neurochir (Wien). Sep 2019;161(9):1887-1894.

6. Millar LM, Robinson AG, O'Flaherty MT, Eames N, Johnston N, Heyburn G. Magnetic resonance imaging in a patient with a dual chamber pacemaker. Case reports in medicine. 2010;2010:292071.

7. Bell DA, Collie D, Statham PF. Cauda equina syndrome: what is the correlation between clinical assessment and MRI scanning? British journal of neurosurgery. Apr 2007;21(2):201-203.

8. Fuso F.A.F., Dias A.L.N., Letaif O.B., Cristante A.F., Marcon R.M., de Barros Filho T.E.P. Epidemiological study of cauda equina syndrome. . Acta ortopedica brasileira. 2013,21(3):159.

9. Adam D, Hornea I. Cauda equina syndrome secondary to lumbar disc herniation. Report of three cases. Romanian Neurosurgery. 2013;3:258-263.

10. Garfin SR, Rydevik BL, Brown RA. Compressive neuropathy of spinal nerve roots. A mechanical or biological problem? Spine (Phila Pa 1976). Feb 1991;16(2):162-166.

11. Rydevik B, Brown MD, Lundborg G. Pathoanatomy and pathophysiology of nerve root compression. Spine (Phila Pa 1976). Jan-Feb 1984;9(1):7-15. 
12. Lee SK, Wolfe SW. Peripheral nerve injury and repair. The Journal of the American Academy of Orthopaedic Surgeons. Jul-Aug 2000;8(4):243-252.

13. Sekiguchi M, Kikuchi S, Myers RR. Experimental spinal stenosis: relationship between degree of cauda equina compression, neuropathology, and pain. Spine (Phila Pa 1976). May 15 2004;29(10):1105-1111.

14. Gitelman A, Hishmeh S, Morelli BN, et al. Cauda equina syndrome: a comprehensive review. Am J Orthop. 2008;37(11):556-562.

15. Al-Badri A., Mbabuike J., Porter DA., Ferriter PJ., Mandel S. Cauda Equina Syndrome: A Case Report and Review of the Literature. Practical neurology. 2013:33-36.

16. Akbar A, A. M. Lumbar disc prolapse: management and outcome analysis of 96 surgically treated patients. . J Pak Med Assoc. 2002;52(2):62-65.

17. Song H., Song Q., Sun C., Yu L., Wang Z., Li Y. Early surgery predicts a better prognosis of urinary function in cauda equina syndrome with retention: a systematic review and meta-analysis. 9. 2016;2(544-551).

18. Dinning TA, Schaeffer HR. Discogenic compression of the cauda equina: a surgical emergency. The Australian and New Zealand journal of surgery. Dec 1993;63(12):927-934.

19. Nielsen B, de Nully M, Schmidt K, Hansen RI. A urodynamic study of cauda equina syndrome due to lumbar disc herniation. Urologia internationalis. 1980;35(3):167-170.

20. Hellström P, Kortelainen P, Kontturi M. Late urodynamic findings after surgery for cauda equina syndrome caused by a prolapsed lumbar intervertebral disk. The Journal of urology. Feb 1986;135(2):308-312.

21. Shapiro S. Cauda equina syndrome secondary to lumbar disc herniation. Neurosurgery. May 1993;32(5):743-746; discussion 746-747.

22. Delgado-López PD, Martín-Alonso J, Martín-Velasco V, et al. Cauda equina syndrome due to disk herniation: Long-term functional prognosis. Neurocirugia (Astur). 2019 Nov - Dec 2019;30(6):278287.

23. Heyes G, Jones M, Verzin E, McLorinan G, Darwish N, Eames N. Influence of timing of surgery on Cauda equina syndrome: Outcomes at a national spinal centre. Journal of orthopaedics. Mar 2018;15(1):210-215.

24. Reddy AP, Mahajan R, Rustagi T, Chhabra HS. Bladder Recovery Patterns in Patients with Complete Cauda Equina Syndrome: A Single-Center Study. Asian spine journal. Dec 2018;12(6):981-986.

25. Lai X-W, Li W, Wang J-X, Zhang H-J, Peng H-M, Yang D-H. Delayed decompression for cauda equina syndrome secondary to lumbar disc herniation: long-term follow-up results. Nan Fang Yi Ke Da Xue Xue Bao. 2017;37(9):1143-1148.

26. Mugge L, Caras A, Miller W, Buehler M, Medhkour A. A Successful Outcome Despite Delayed Intervention for Cauda Equina Syndrome in a Young Patient with a Posterior Epidural Disc Extrusion. Cureus. May 11 2019;11(5):e4645. 
27. Douraiswami B, Muthuswamy K, Naidu DK, Thanigai S, Anand V. Indeterminate cauda equina syndrome: A case report. Journal of clinical orthopaedics and trauma. Jan-Mar 2016;7(1):50-54.

\section{Figures}

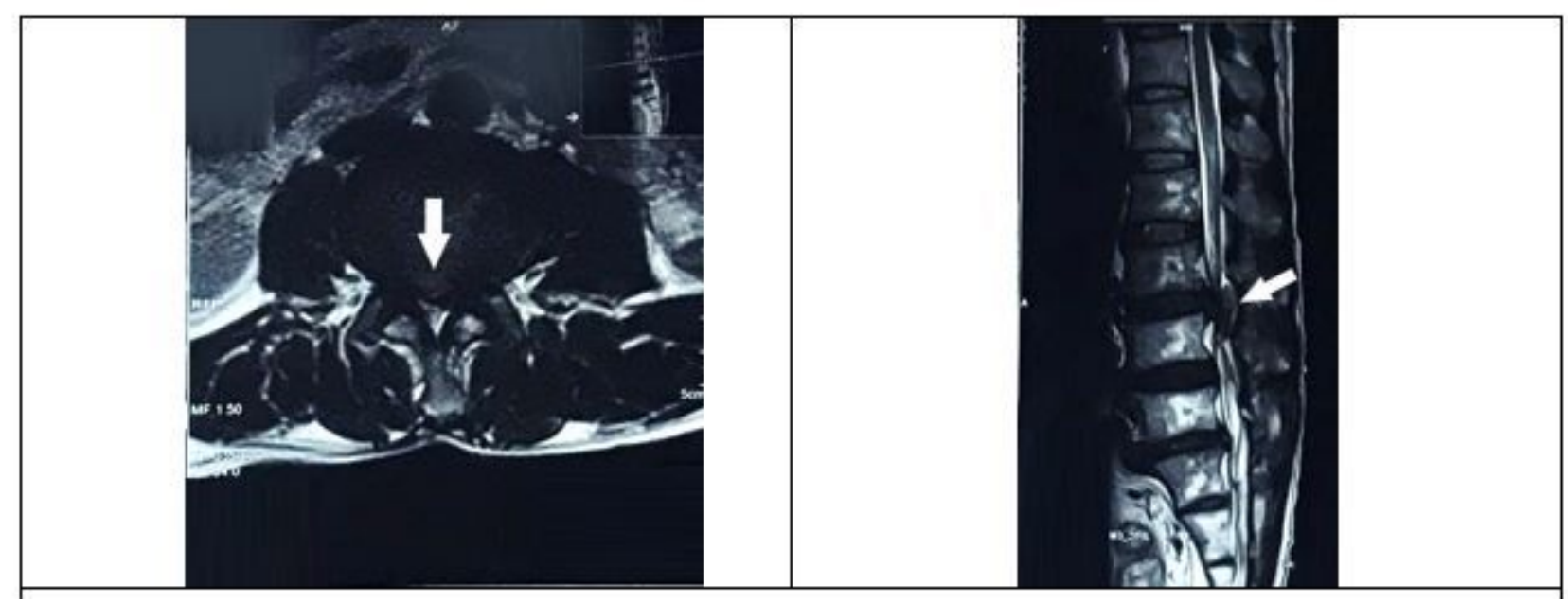

\section{Figure 1}

At the level of L3-L4, there is a larger herniation downward, causing severe narrowing of the spinal canal (degree $\mathrm{C}$ ), the foraminal zone on both sides, and compression of the cauda equina.

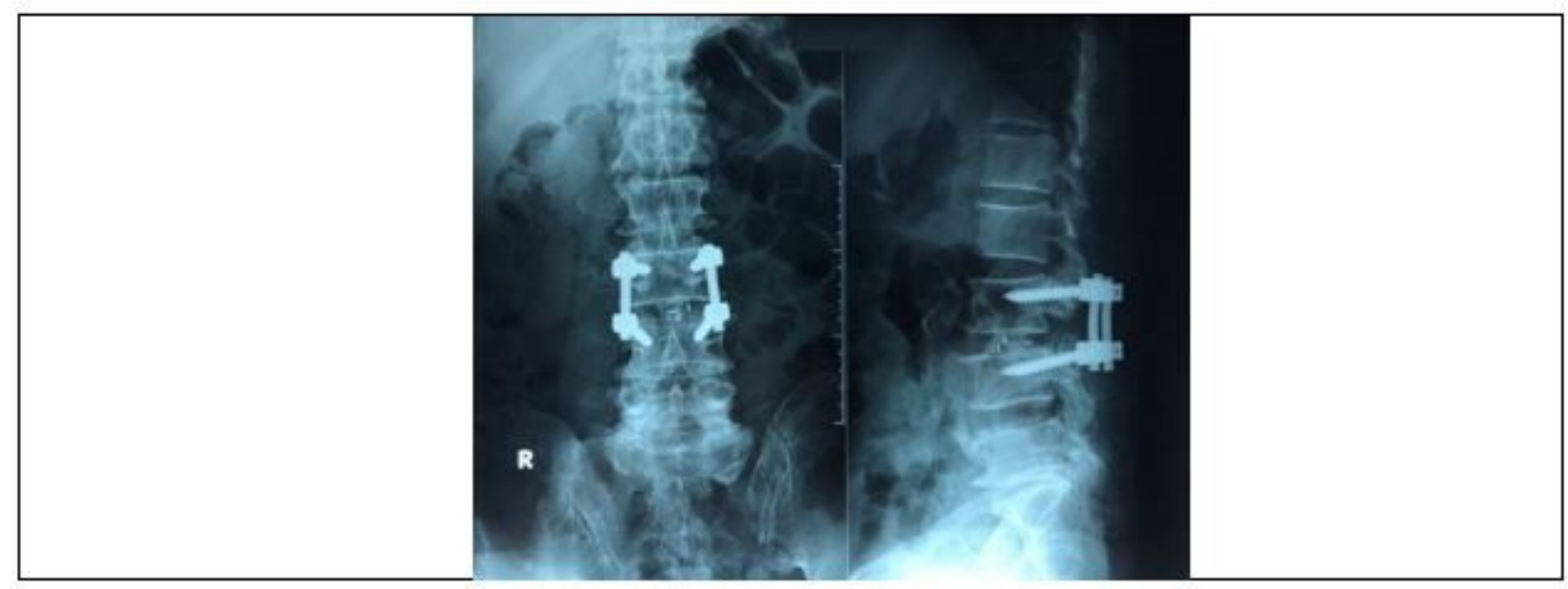

\section{Figure 2}

Laminectomy and decompression at the L3-L4 level using the posterior lumbar interbody fusion (PLIF) procedure 


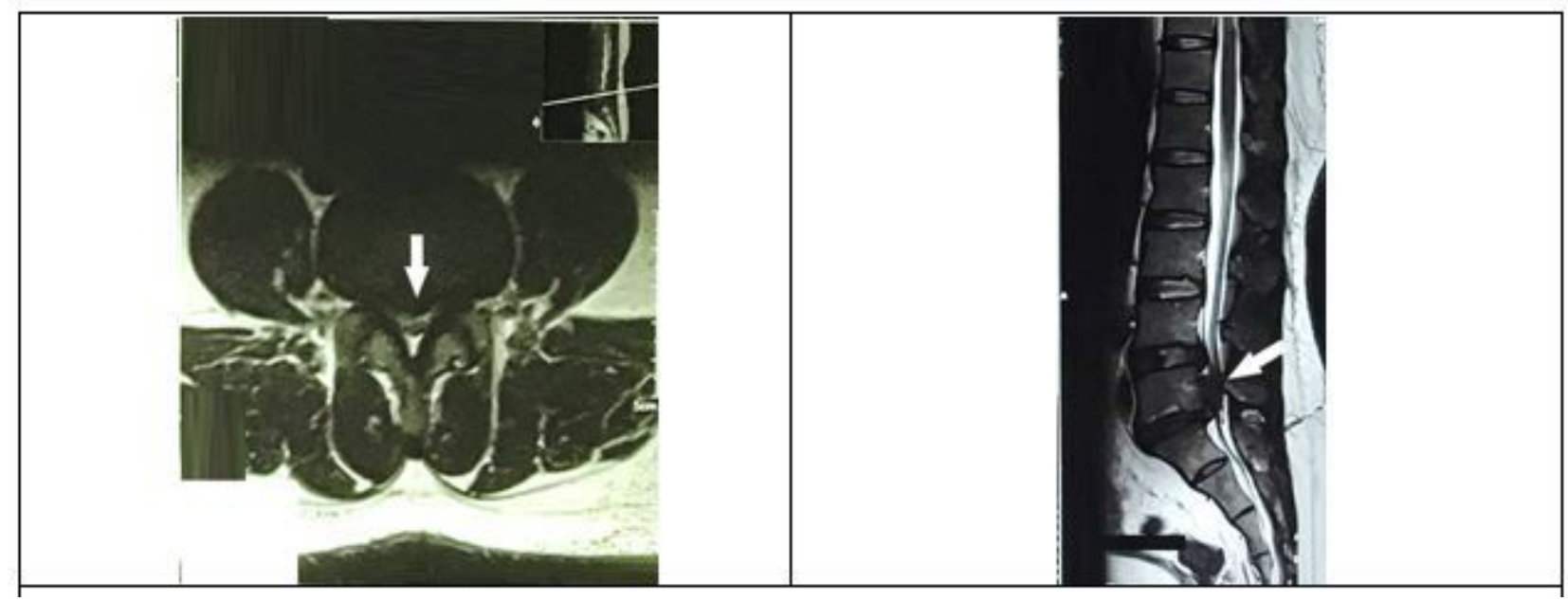

\section{Figure 3}

MRI showed an extruded disc herniation at the L5- S1, and a posterior protrusion at L4- L5 resulting in central canal stenosis and compression of the cauda equina

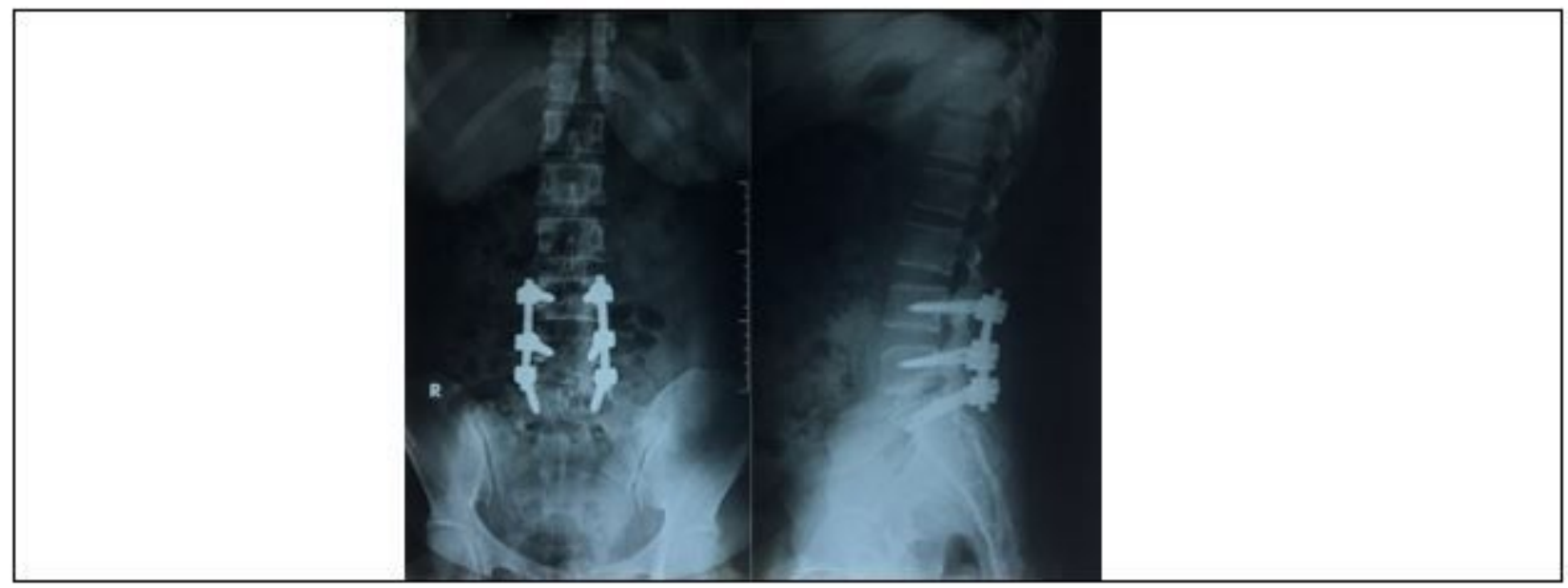

Figure 4

Laminectomy and an adequate width decompression at the L4-L5, L5-S1 level using the posterior lumbar interbody fusion (PLIF) procedure 